rheuma plus $2018 \cdot 17: 145$

https://doi.org/10.1007/s12688-018-0213-z

(c) Springer-Verlag GmbH Austria, ein Teil von

Springer Nature 2018

\section{Burkhard Leeb}

Institut für Klinische Rheumatologie der Karl Landsteiner Gesellschaft, Hollabrunn, Österreich

\title{
Rheuma kommt selten allein
}

\section{Liebe Leserinnen und Leser!}

Der Sommer neigt sich unweigerlich seinem Ende zu. Egal welchen Platz er in der Hitliste der Klimakatastrophen-Propheten einnehmen wird, heiß war's und wärmer als 1975, denn da sang Rudi Carell noch: „Wann wird's mal wieder richtig Sommer?" Nun aber geht dieser „haaße Summer" (O-Ton Reinhard Fendrich 1982) zu Ende. Auf der anderen Seite liefert aber das Wetter, vor allem die Feuchtigkeit, häufig eine Ausrede für Verschlechterungen von rheumatischen Beschwerden, größtenteils völlig ohne Evidenz. Wetterfühligkeit ist nur ein, und nicht das wesentlichste Begleitsymptom, bei rheumatischen Erkrankungen. In der Praxis stellt, vor allem mit steigendem Alter, die rheumatische Erkrankung nicht das einzige gesundheitliche Problem der Patienten dar.

\section{Wirft Multimorbidität alles über den Haufen?}

Wirft Multimorbidität die heile artifizielle Welt der Empfehlungen, Richtlinien, points to consider und ähnlicher Elaborate über den Haufen? Evidenzbasierte Empfehlungen beziehen sich immer nur auf eine Erkrankung. Es ist verständlich, dass Begleiterkrankungen außer Acht gelassen werden müssen, mit einer Ausnahme: Es wird üblicherweise angemerkt, dass im Falle von Begleiterkrankungen man den entsprechenden Empfehlungen folgen solle.

Ein kleines Beispiel: Die allermeisten Empfehlungen zur Therapie der Arthrose beinhalten nicht-steroidale Antirheumatika oder wenigstens Paracetamol. Führt man sich aber die am häufigsten von Arth- rose Betroffenen vor Augen, so sind diese meist älter als 65 Jahre, haben häufig eine arterielle Hypertonie oder eine KHK, eine Nierenfunktionseinschränkung etc. Man lese dazu dann die entsprechenden Empfehlungen. Wer bleibt in dieser sicher redlich geführten Debatte übrig? Der schmerzgeplagte Patient, aber das ist Theoretikern ja nicht so wichtig.

Der Ausweg aus diesem Dilemma: Leitlinien für Multimorbidität. Die gibt es aber in viel geringerer Zahl als die „einfachen" Empfehlungen, denn die klinischen Studien, auf denen die evidenzbasierten Empfehlungen beruhen schließen meist multimorbide Patienten aus und nicht für alle Erkrankungen existieren Register, um real world data zu generieren. So fand eine rezente Cochrane Review über Interventionen spezifisch bei multimorbiden $\mathrm{Pa}$ tienten lediglich zehn randomisierte Studien weltweit.

Empfohlen wird dann, man glaubt es kaum, z. B. 2016 seitens der WHO, dass es potenzielle Interaktionen zwischen Medikamenten und auch zwischen Erkrankungen gefährlich machen können, Empfehlungen, die nur auf eine Krankheit abzielen, bei Patienten mit mehreren Erkrankungen anzuwenden. Es lebe der exklusiv rheumatisch Erkrankte!

Selbstverständlich existieren auch für die Gicht Leitlinien und Empfehlungen - und zwar, oh Graus, nicht nur von Rheumatologen, sondern auch von $\mathrm{Ne}$ phrologen, Hypertensiologen, Endokrinologen und Kardiologen, mit zum Teil durchaus diskrepantem Inhalt. Ja auch die Gicht kommt selten allein (Schlagwort metabolisches Syndrom). Vielleicht lässt sich dieses Dilemma ja auch durch persönliche, möglicherweise divergente, Expertise lösen. Die Rheumatologen
Gabriele Eichbauer-Sturm, Linz, auch Nephrologin, und Gregor Holak, Wien, auch Stoffwechselexperte, beleuchten in Pro- und Contra-Statements die Indikationsstellung zur Harnsäure-senkenden Therapie.

Eine weitere Übersicht von Martin Rudwaleit, Bielefeld, beschäftigt sich mit einer unangenehmen Komplikation von Spondyloarthritiden, nämlich mit der Uveitis.

Wir hoffen, auch mit dieser Ausgabe wieder Ihr Interesse wecken zu können, um dem Ziel von rheuma plus nahe zu kommen, eine fruchtbringende, offene und redliche Diskussion in Gang zu bringen, aus der alle Nutzen ziehen können. Wie immer an dieser Stelle möchten wir Sie herzlich dazu einladen, uns Ihre Meinung zu den Beiträgen dieser Ausgabe, wie auch zu allen rheumatologischen Themen, die Sie für relevant oder für diskussionswert halten, zu schicken. Sie können in jedem Fall auf einen fairen Review mit entsprechendem Feedback vertrauen. Für Kritik, Hinweise, Zustimmung wie auch für jeden anderen Beitrag bedanke ich mich schon im Voraus.

Herzlichst,

Ihr Burkhard Leeb

\section{Korrespondenzadresse}

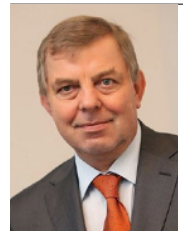

Burkhard Leeb

Babogasse 20, 2020 Hollabrunn, Österreich leeb.rheuma@aon.at www.leeb-rheuma.at 